A) Check for updates

Cite this: Analyst, 2020, 145, 6937

\title{
A multilayered cancer-on-a-chip model to analyze the effectiveness of new-generation photosensitizers $\dagger$
}

\author{
Magdalena Flont, (D) Elżbieta Jastrzębska (D) and Zbigniew Brzózka (D)*
}

Three-dimensional (3D) cellular models of cancer tissue are necessary tools to analyze new anticancer drugs under in vitro conditions. Diagnostics and treatment of ovarian cancer are major challenges for current medicine. In our report we propose a new three-dimensional (3D) cellular model of ovarian cancer which can mimic a fragment of heterogeneous cancer tissue. We used Lab-on-a-chip technology to create a microfluidic system that allows cellular multilayer to be cultured. Cellular multilayer mimics the structure of two important elements of cancer tissue: flesh and stroma. For this reason, it has an advantage over other in vitro cellular models. We used human ovarian fibroblasts (HOF) and human ovarian cancer cells in our research (A2780). In the first stage of the study, we proved that the presence of nonmalignant fibroblasts in co-culture with ovarian cancer cells stimulates the proliferation of cancer cells, which is important in the progression of ovarian cancer. In the next stage of the research, we tested the usefulness of the newly-developed cellular model in the analysis of anticancer drugs and therapies under in vitro conditions. We tested two photosensitizers (PS): free and nanoencapsulated meso-tetrafenylporphyrin, and we evaluated the potential of these drugs in anticancer photodynamic therapy (PDT) of ovarian cancer. We also studied the mechanism of PDT based on the analysis of the level of reactive oxygen species (ROS) in cell cultures. Our research confirmed that the use of new-generation PS can significantly increase the efficacy of PDT in the treatment of ovarian cancer. We also proved that the newlydeveloped 3D cellular model is suitable for rapid screening of anticancer drugs and has the potential to be used clinically in the future, e.g. in the selection of treatment methods for anticancer personalized medicine.

Received 6th May 2020, Accepted 16th August 2020 DOI: $10.1039 / \mathrm{d} 0$ an00911c rsc.li/analyst

\section{Introduction}

Laboratory studies on the newly developed anti-cancer compounds are often performed on simple two-dimensional (2D) cellular models. Developing the three-dimensional (3D) tissue models, that mimic specificity of a living organism is one of the main goals of cellular engineering today. ${ }^{1,2}$ Under physiological conditions, the structure of a tumor tissue is composed of both tumor (parenchyma) and non-malignant cells (stroma). Stroma consists mainly of blood vessels and connective tissue cells (e.g. fibroblasts). ${ }^{3,4}$ Stromal cells perform supporting, integration and nutritional functions for cancer cells. $^{{ }^{-7}}$ Tumor-stroma interactions are multifactorial molecular mechanisms and key elements in cancer biology. ${ }^{8}$ They

Chair of Medical Biotechnology, Faculty of Chemistry, Warsaw University of Technology, Noakowskiego 3, 00-664 Warsaw, Poland. E-mail: brzozka@ch.pw.edu.pl ; Tel: +48222345427

$\dagger$ Electronic supplementary information (ESI) available. See DOI: 10.1039/ d0an00911c are responsible for the progression or inhibition of tumor growth, but can also affect the tumor's response to treatment. ${ }^{9}$ Thus, the cancer microenvironment may affect the effectiveness of anti-cancer drugs. ${ }^{10}$ In response to treatment, stromal cells may induce more intensive production of growth factors in cancer cells or regulate the various anti-apoptotic signaling pathways that were disrupted by treatment (e.g. pathways responsible for DNA repair). Drug resistance mediated by the tumor microenvironment is not applicable only to conventional therapies, e.g. chemotherapy, but is characteristic of a wide spectrum of anti-cancer compounds. ${ }^{9,11}$ Therefore, the stroma should be included in cancer in vitro modeling to avoid confusing effects in anti-cancer drug screening. The in vitro cancer cell culture conditions can be modified to better mimic the conditions of physiological tumor growth. ${ }^{12}$ Co-culture of cancer cells and non-malignant cells of connective tissue (e.g. fibroblasts) to a certain extent can mimic the structure of a tumor. $^{13-15}$

A modern way to create 3D cell cultures is to use Lab-on- $a$ chip microfluidic systems that allow cells to be grown in a 
culture under dynamic conditions. ${ }^{16}$ It was proved that in vitro stimulation of cells with a medium flow promotes the creation of $3 \mathrm{D}$ cellular structures with layered cell arrangements. ${ }^{14}$ In addition, the use of the appropriate flow rate in the microsystem reflects the in vivo conditions of the flow of bodily fluids. ${ }^{17-20}$ However, it is necessary to design the appropriate geometry of the microsystem to ensure good conditions for 3D cell culture. In the last few years, more microsystems have been used in cellular engineering, but the microdevices to create effective 3D cell models are still to be developed.

Ovarian cancer is a serious problem because of its the high mortality rate. ${ }^{21}$ It is difficult to treat ovarian cancer because it often metastasizes to other organs, especially in the abdominal cavity. Cytoreductive surgery followed by chemotherapy based on carboplatin, paclitaxel and doxorubicin is the standard therapeutic treatment for ovarian cancer. ${ }^{22}$ Mimicking the physiological structure of ovarian cancer under laboratory conditions may allow rapid testing of the effectiveness of new drugs and therapies in the treatment of this type of cancer. Photodynamic therapy (PDT) is a new approach to the treatment of ovarian cancer. ${ }^{23,24}$

PDT is a minimally invasive method for cancer treatment that uses light-sensitive drugs (photosensitizers, PS). ${ }^{25}$ PS are compounds that have high chemical purity and selectively target cancer cells. They are characterized by the ability to ability to intensively form reactive oxygen species in tissues. The best studied group of PS are porphyrins and their derivatives, which belong to the first and second generation of PS. There is also a new, third generation of PS, which includes second-generation photosensitizers coupled with molecular carriers (e.g. encapsulated in nanocapsules). ${ }^{26,27}$ In recent years, it was proved that nanoencapsulation of PS can significantly improve their photodynamic properties. The nanoencapsulation process may improve the solubility of poorly watersoluble drugs, increase the selectivity of PS delivery to target cells and reduce the problem of PS photodegradation. Controlled release of the drug in the cells is also possible thanks to the use of nanocarriers. ${ }^{28}$ Due to the growing number of new-generation PS, which are based mainly on nanoparticles, PDT has been showing an increased potential for use as cancer treatment. ${ }^{29}$

In recent years, the high effectiveness of PDT in supporting chemotherapy or surgical treatment of ovarian cancer was clinically confirmed. ${ }^{30,31}$ Allison et al. described cases in which ovarian cancer and peritoneal metastases were treated with PDT. Patients received PS (e.g. Photofrin, Foscan) intravenously. An intraoperative irradiation with safe, green light $(514 \mathrm{~nm})$ was then performed. The use of high-power intraoperative irradiation can cause side effects. ${ }^{32}$ However, research to improve the irradiation stage is being carried out, e.g. by increasing the precision of irradiation through the use of laparoscopy. The application of modern optical fibers can allow the use of high-powered and long wavelength light and reduces the side effects of treatment. ${ }^{24,33}$ Azaiis et al. confirmed that in recent years intensive in vivo research on the use of PDT in the treatment of ovarian cancer residues after cytore- ductive surgery has been conducted. ${ }^{22}$ More extensive research on early-stage ovarian cancer treatment with PDT may be of key clinical importance, especially since ovarian cancer is one of the most invasive cancers of reproductive organs. ${ }^{24,25,34,35}$

In our report, we have decided to create a new, 3D ovarian cancer model, using a Lab-on-a-chip microsystem. We assumed, that the proposed cellular model can imitate a fragment of structure of heterogeneous cancer tissue. We decided to check whether the mimicking of the tumor stroma under microfluidic conditions would stimulate cancer cells to more intensive proliferation. In addition, we hypothesized that a new research model (multilayer) would be a good tool for rapidly screening anticancer drugs and for testing anticancer therapies under in vitro conditions. For this purpose, we tested two photosensitizing drugs in the developed microsystem, free and nanoencapsulated meso-tetrafenylporphyrin. We used a new cellular model and we checked and compared the effectiveness of tested PS in PDT on ovarian cancer. We also tested the correctness of the nanoencapsulated drug's mechanism of action, based on the level of ROS.

\section{Materials and methods}

\section{The microfluidic system design and fabrication}

In our research, we developed a new microfluidic system for three-dimensional (3D) multilayered cell culture. The microsystem was made of two biocompatible and transparent materials: poly(dimethylsiloxane) (PDMS) and glass. The microsystem was equipped with six main microchambers. Each of them contained a circular microwell, with a diameter of $2 \mathrm{~mm}$ and a depth of $100 \mu \mathrm{m}$. The microwell was the target place of cell culture. The longitudinal extension of the microchannel as a space filled with culture medium was placed above the microwell. Longitudinal extention of microchannels also enabled precise introduction of the cells into the microwells. The microchambers were connected by a network of microchannels with a depth of $200 \mu \mathrm{m}$ and a width of $100 \mu \mathrm{m}$. The microchannel network was initiated by two independent inlets (with supplying tubes) and ended with one common outlet (with outflowing tube) (Fig. 1A). This solution enabled one-step analysis of both a drug and control samples on cells cultured in one microsystem.

The microsystem geometry was three-dimensional. A 3D structure of the microsystem geometry was achieved thanks to the hybrid combination of three layers of construction materials: (1) a PDMS layer (with a thickness of $500 \mathrm{~mm}$ ) equipped with a microchannel network, (2) a thin layer of PDMS (with a thickness of $100 \mu \mathrm{m}$ ) equipped with six holes with a diameter of $2 \mathrm{~mm}$ and (3) a layer of sodium glass with dimensions: $76 \mathrm{~mm} \times 25 \mathrm{~mm} \times 1 \mathrm{~mm}$ (Fig. $1 \mathrm{~A}$ and B). All elements of the microsystem were permanently connected to each other using an oxygen plasma generator (Diener Electronic Atto).

Human ovarian fibroblasts are adherent cells, but under in vitro conditions they can easily detach from the growth sub- 


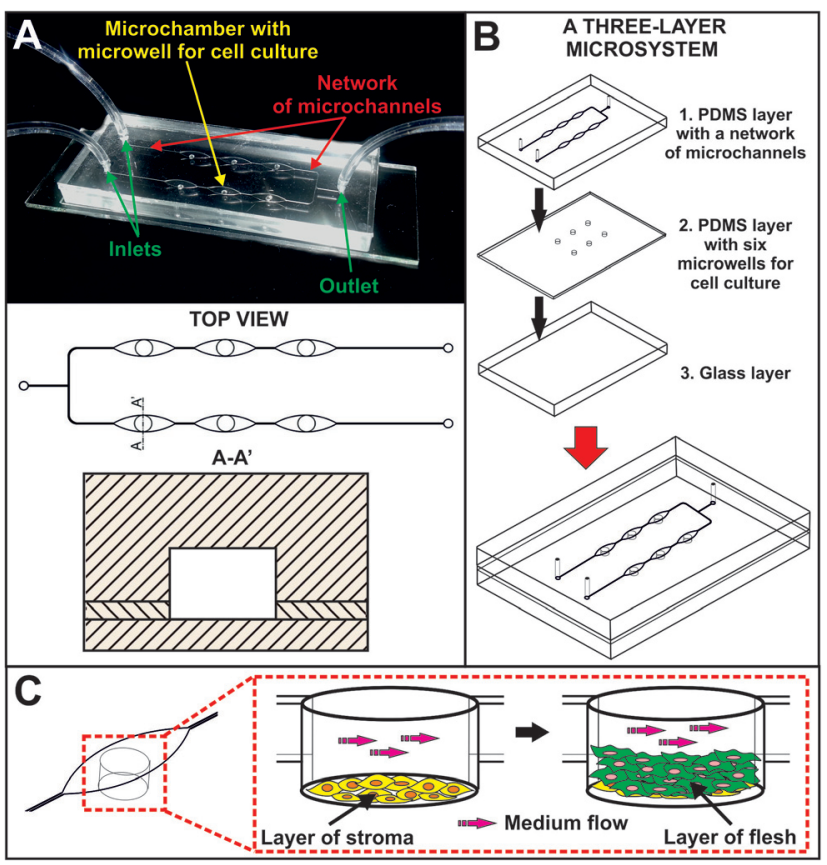

Fig. 1 (A) A geometry of the designed microsystem and cross-section of the microchamber for cell culture. (B) A scheme of three-dimensional (3D) structure of the microsystem. (C) The idea of using a microwell for non-malignant cell culture (stroma) and for controlled formation of cancer cell multilayer (flesh).

strate because of mechanical factors. A direct exposure to flow can cause HOF cells to detach and be removed from the microchamber. The use of microwells in the microsystem reduced the chance of the cells detaching from the substrate and allowed hydrodynamic stress inside the microsystem to be minimized. In addition, the application of the microwell allowed for precise control of the multilayered cell culture formation (Fig. 1C).

There was a linear, double row distribution of microchambers for the cell culture, it corresponded to the distribution of wells on a standard 384-well plate. Thanks to this, it was possible to carry out tests on the cultured cells using spectrofluorimetric detection. Adaptation of the microsystem to the multiwell plate reader (Varian) enabled rapid, automatic spectrofluorimetric measurements.

The network of microstructures was patterned in the upper layer of PDMS (1) using a micromilling technology and the replica molding technique. A detailed description of these techniques was presented in our previous works. ${ }^{36,37}$ A thin layer of PDMS (2) was made by casting a non-crosslinked PDMS between two flat poly(methyl methacrylate) (PMMA) plates. The distance between the plates was $100 \mu \mathrm{m}$. Holes (corresponding to microwells) in the PDMS thin layer (2) were made using a precise Uni-Core point punch.

\section{Cell lines}

Two ovarian cell lines: non-malignant and cancerous were used in this research. The non-malignant cell line was human ovarian fibroblasts (HOF), obtained from ScienCell Research Laboratories. HOF are connective tissue cells that derive from the mesoderm. The cancer cell line was human ovarian carcinoma (A2780), obtained from American Type Culture Collection. The non-malignant cells were cultured using $75 \mathrm{~cm}^{2}$ standard culture flasks in DMEM medium (Sigma) supplemented with 10\% vol. fetal bovine serum (Gibco), 1\% vol. streptomycin and penicillin (Sigma), $2 \mathrm{mM}$ L-glutamine (Sigma) and 1\% vol. Fibroblast Growth Supplement (Sigma). The cancer cells were cultured using $25 \mathrm{~cm}^{2}$ standard culture flasks in RPMI medium (Sigma) supplemented with 10\% vol. fetal bovine serum (Gibco, thermally inactivated), 1\% vol. streptomycin and penicillin (Sigma), and $2 \mathrm{mM}$ L-glutamine (Sigma). Both cell lines were cultured at $37{ }^{\circ} \mathrm{C}$ in a humidified atmosphere including $5 \% \quad \mathrm{CO}_{2} \quad$ (HeraCell 150, Thermo Scientific). During the tests in micro- and macroscale, nonmalignant cells and cancer cells grew independently (as monoculture) or together (as co-culture).

\section{Cell culture formation under microfluidic conditions}

Before the experiment, the microsystem was sterilized using a $70 \%$ vol. ethanol solution and UV light (15 min exposure). Then, the microsystem was filled with DMEM culture medium (Sigma), and the inlet and outlet tubes were sealed. To increase the cell adhesion, the microsystem was filled in and incubated with culture medium $\left(37^{\circ} \mathrm{C}, 5 \% \mathrm{CO}_{2}\right)$ for a minimum of $2 \mathrm{~h}$. In the first step, to create a cellular multilayer, a suspension of the non-malignant cells (HOF) was prepared. For this purpose, the cells were washed with phosphate buffer without calcium and magnesium ions (DPBS, Thermo Fisher), and then were detached from the culture bottle substrate with Trypsin (Sigma). The cell suspension was centrifuged to concentrate it to density of $10^{6}$ cell per mL. HOF cells were introduced into the microsystem to cover approximately $90 \%$ of the surface of each culture microwell. The cells were introduced using a peristaltic pump (Ismatec Reglo-Digital

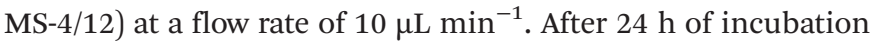
$\left(37{ }^{\circ} \mathrm{C}, 5 \% \mathrm{CO}_{2}\right)$, when the non-malignant cells were adhered to the glass substrate, a suspension of cancer cells was prepared (A2780). For this purpose, the A2780 cells were washed with phosphate buffer (PBS, Thermo Fisher) and peeled off the substrate with Trypsin (Sigma). A cell suspension with density of $3 \times 10^{6}$ cell per $\mathrm{mL}$ was prepared. Cancer cells were introduced into the microsystem in the same way as non-malignant cells. The microsystem with the cells was incubated $\left(37^{\circ} \mathrm{C}, 5 \%\right.$ $\mathrm{CO}_{2}$ ) for the next $24 \mathrm{~h}$. After this time, the formation of a cellular multilayer was monitored using microscopic observation. The monolayer cultures of the non-malignant and cancer cells were also performed in the designed microsystem as an independent control. For this purpose, each of the cell lines was separately introduced into the microsystem, according to the procedure described above.

To confirm the multilayered arrangement of A2780 and HOF cells in the microsystem a confocal scanning laser microscope (FLUOVIEW FV10i-LIV Confocal Laser Scanning Microscope, Olympus) was also used. Cell culture analysis was 
performed with the ability to scan the view in three dimensions. For this purpose, $170 \mu \mathrm{m}$ thin glass was used in the construction of the microsystem. The cells were introduced into the microsystem and stained with fluorescent dyes: propidium iodide (concentration of stock solution: $1 \mathrm{mg} \mathrm{mL}^{-1}$ ) and Calcein-AM (concentration of stock solution: $2 \mathrm{mM}$ ). The cells were incubated with a solution of dyes $\left(37^{\circ} \mathrm{C}, 5 \% \mathrm{CO}_{2}\right)$ for $10 \mathrm{~min}$. After incubation, a $Z$-axis scan of cell culture was performed using a confocal microscope.

\section{Analysis of the cell proliferation in a multilayered cell culture}

The AlamarBlue test was used to check the proliferation of $\mathrm{HOF} / \mathrm{A} 2780$ cell co-culture (growing in the form of a cellular multilayer) as well as the HOF and A2780 cell mono-cultures (growing in the monolayer). For this purpose, the AlamarBlue reagent solution (BioRad) with concentration of $10 \%$ vol. was prepared in the culture medium daily and introduced into the microsystem for $15 \mathrm{~min}$ at a flow rate of $2 \mu \mathrm{L} \mathrm{min}^{-1}$. The microsystem was incubated for $45 \mathrm{~min}\left(37{ }^{\circ} \mathrm{C}, 5 \% \mathrm{CO}_{2}\right)$ and afterwards, fluorescence intensity was measured $\left(\lambda_{\mathrm{ex}}=552 \mathrm{~nm}\right.$, $\lambda_{\text {em }}=582 \mathrm{~nm}$ ), using a multi-well plate reader (Varian). The measured fluorescence intensity was assumed to be directly proportional to the number of proliferating cells in the culture microchamber. Based on the results of the proliferation tests, we could check how the presence of basal cells (fibroblasts) affects the intensity of cancer cell division. As a result, it was possible to confirm or exclude the formation of a multilayered cell culture in the microsystem.

\section{Photodynamic therapy (PDT) procedure}

The developed microfluidic system was used to assess the cytotoxicity and photocytotoxicity of a photosensitizing compound (photosensitiser, PS) - meso-tetrafenylporphyrine. mesoTetrafenylporphyrin was tested in two forms: free (TPP) and nanoencapsulated (nano-TPP). The free form of the tested drug is a synthetic heterocyclic porphyrin derivative and is commercially available (Sigma). The nanoencapsulated form of the drug was synthesized using the layer-by-layer (LbL) saturation method. The oil-core polyelectrolyte TPP-loaded nanocapsules with (PSS/PDADMAC) ${ }_{4}$ shell and stabilized by a dicphalic-type surfactant $\mathrm{C}_{12} \quad(\mathrm{TAPAMS})_{2}$ were prepared. The details of the synthesis and physico-chemical properties of the nanoencapsulated photosensitizer were presented in our previous works. ${ }^{38,39}$

In order to carry out the photodynamic therapy (PDT) procedure, the solutions of tested PSs at concentrations of 0-30 $\mu \mathrm{M}$ were prepared in the DMEM culture medium (without phenol red). $24 \mathrm{~h}$ after the introduction of the HOF and A2780 cells into the microsystem, the PS solutions were

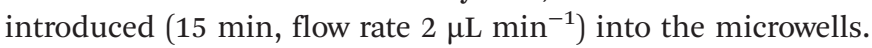
The cells were incubated with PS in the dark $\left(37{ }^{\circ} \mathrm{C}, 5 \% \mathrm{CO}_{2}\right)$ for $24 \mathrm{~h}$. This is the optimal time to penetrate the PS inside the cells. ${ }^{40-42}$ After this time, the AlamarBlue viability assays were performed to determine the cytotoxic properties of PS. Then, the cells with the accumulated PS were irradiated. For this purpose, a LED light source with a power of $40 \mathrm{~mW} \mathrm{~cm}-2$ and a wavelength of $640 \mathrm{~nm}$ was used. The cells were irradiated for $10 \mathrm{~min}$ at a distance of $10 \mathrm{~mm}$ from the light source. $24 \mathrm{~h}$ after the irradiation, the viability tests were again performed to determine the photocytotoxicity of PS.

\section{Analysis of cell viability in the microsystem - test AlamarBlue and test CAM/PI}

To determine the cell viability in the microsystem after incubation with the photosensitizer and after the PDT procedure, two independent tests were used: AlamarBlue and differential staining with Propidium iodide (PI, Sigma) and Calcein-AM (CAM, Sigma). The AlamarBlue test was performed in the same way as in the analysis of cell proliferation. Differential staining of live and dead cells, using fluorescent dyes, was performed for qualitative results analysis. For this purpose, a mixture of: $1 \mu \mathrm{l}$ of PI (concentration of stock solution: $1 \mathrm{mg} \mathrm{mL}^{-1}$ ), $1 \mu \mathrm{l}$ of CAM (concentration of stock solution: $2 \mathrm{mM}$ ) and $500 \mu \mathrm{l}$ of culture medium was introduced into the microsystem and incubated $\left(37^{\circ} \mathrm{C}, 5 \% \mathrm{CO}_{2}\right)$ for $10 \mathrm{~min}$. After this time, CAM penetrated into living cells and they showed green fluorescence. PI penetrated into dead cells, causing them to give red fluorescence. The test results were observed using an inverted fluorescence microscope (Olympus IX71).

\section{Analysis of the level of reactive oxygen species (ROS)}

The study of the level of reactive oxygen species (ROS) was carried out in order to analyze the mechanism of PDT in the monoculture and coculture of non-malignant and cancer ovarian cells. Non-malignant and cancer cells were seeded on a standard 96-well culture plate and cultured in the form of monoculture or co-culture. When the cells were adherent, a solution of nano-TPP $(5 \mu \mathrm{M})$ or the culture medium $(0 \mu \mathrm{M})$ as a control was added. After $24 \mathrm{~h}$ of cell incubation with PS $\left(37{ }^{\circ} \mathrm{C}, 5 \% \mathrm{CO}_{2}\right.$ ), the ROS level was determined. For this purpose, a $10 \mathrm{mM}$ solution of the oxidant-sensitive dye $2^{\prime}, 7^{\prime}$ dichlorofluorescin diacetate (DCFDA, Sigma) was prepared: $0.485 \mathrm{mg}$ of DCFDA was weighed and dissolved in $100 \mu \mathrm{l}$ of dimethyl sulfoxide (DMSO, Sigma). The solution was diluted in the culture medium without serum and phenol red to a concentration of $20 \mu \mathrm{M}$. The cells were washed with PBS and, after that, the DCFDA working solution was added to the cells $\left(100 \mu \mathrm{l}\right.$ per well). The cells were incubated for $30 \mathrm{~min}\left(37^{\circ} \mathrm{C}\right.$, $5 \% \mathrm{CO}_{2}$ ) and then washed again with PBS, $100 \mu \mathrm{l}$ of PBS was added per well and the fluorescence intensity was measured $\left(\lambda_{\text {ex }}=485 \mathrm{~nm}, \lambda_{\text {em }}=520 \mathrm{~nm}\right)$. In the next step, the cells were irradiated (irradiation parameters as in the microscale) and 45 min after irradiation, the fluorescence intensity was again measured.

\section{Data analysis}

In the statistical data analysis, a one-way analysis of variance was used (ANOVA test) where the differentiating factor was the concentration of the tested drug. All differences in data (increase/decrease in value) marked on diagrams with asterisks are statistically significant for the level of significance $\alpha$ set at 0.05 (95\% confidence interval). Each experiment was indepen- 
dently carried out at least three times. All numerical results are expressed as an average of a minimum of three independent measurement repeats \pm standard deviation (SD).

\section{Results}

Three-dimensional imaging multilayered cell culture in the microsystem

A scanning confocal microscope was used to analyze the formation of multilayer co-culture of A2780 and HOF cells in the microsystem. In our experiment, we presented a cross-sectional view of the cell culture in the microsystem (Fig. 2). We showed that there were regions in the cell culture in the microsystem where A2780 and HOF cells grew as cell monolayers. However, the formation of many extensive three-dimensional regions, in which cancer and non-malignant cells grew in the form of multilayer were observed. The images obtained by the scanning confocal microscope showed that the cells in 3D regions had high viability (cells showed green fluorescence) (Fig. 2). It was proved that A2780 and HOF cells can be cultured longterm in the form of a cellular multilayer and, to some extent, mimic a fragment of cancer tissue.

\section{Cell proliferation in a multilayer cellular model}

In our experiments, we cultured the non-malignant and cancer ovarian cells in the microfluidic system in two forms: a cellular monolayer (monoculture) and cellular multilayer (co-culture) (Fig. 3). Under physiological conditions, there are many interactions between stromal and tumor cells. Stroma-tumor interactions play an important role in cancer development. The presence of stromal cells can affect the intensity of cancer cell proliferation. ${ }^{9,15,38}$ The aim of our study was to check whether the presence of non-malignant cells (fibroblasts) in a cancer cell culture can affect the intensity of cancer cells divisions.

Analysis of the HOF cell proliferation showed that within $96 \mathrm{~h}$ of culture under microfluidic conditions, the number of cells in the population did not change significantly. The ovarian non-malignant cells proliferated very slowly and doubling of the number of cells in the population was not
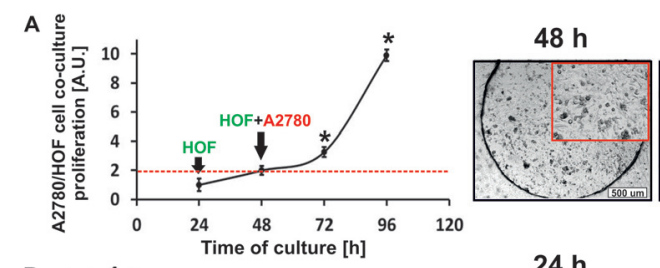

$96 \mathrm{~h}$
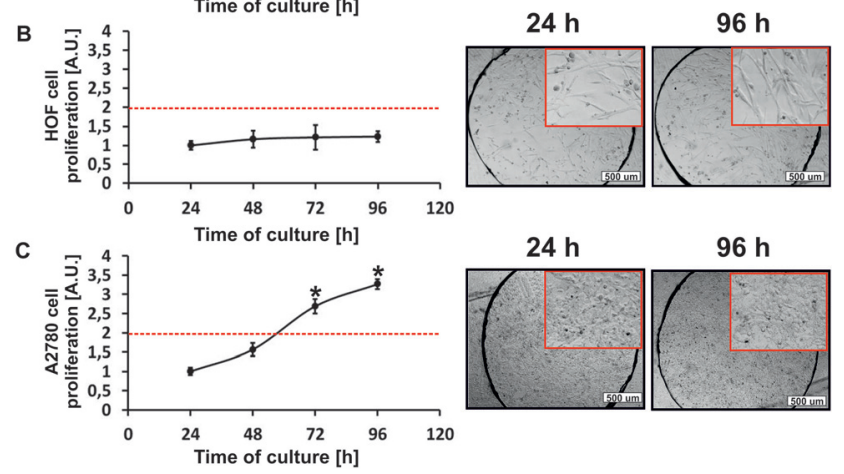

Fig. 3 The intensity of proliferation of non-malignant (HOF) and cancer cells (A2780) cultured under microfluidic conditions (A) in the form of co-culture (cellular multilayer) and (B and $C$ ) in the form of independent monocultures (cellular monolayer). The pictures show changes in cell morphology in the first ( $24 \mathrm{~h}$ or $48 \mathrm{~h}$ ) and last (96 h) day of culture in the microsystem (Olympus IX71). The red line determines the moment of doubling the number of cells in population.

observed (Fig. 3B). In the case of A2780 monoculture, the number of cells in the population on the last day of culture (96 h) increased 3-fold, compared to the first day of culture (24 h) (Fig. 3C). A significant increase in the number of cells means that the ovarian cancer cells cultured under microfluidic conditions were intensively divided. The differences in the rate of divisions between the HOF and A2780 cells may result from their genesis and biological nature. Fibroblasts used in the experiment are connective tissue cell that have much lower speed division than the cancer cells. ${ }^{43}$ Cancer cells are characterized by unlimited divisions. The results confirm that the described cell features are also maintained under the proposed in vitro microfluidic conditions.

The analysis of the proliferation of the HOF/A2780 cell coculture (cultured as a multilayer) showed that the total number

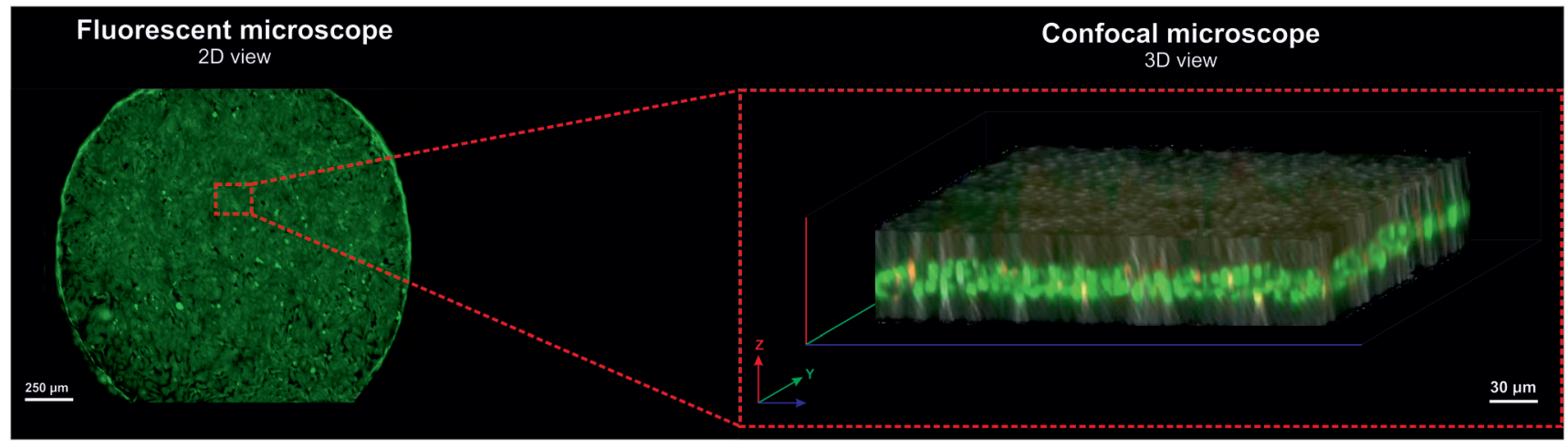

Fig. 2 Three-dimensional imaging of the A2780/HOF cell co-culture on the fourth day of culture. The cells were stained with propidium iodide (red points - dead cells) and Calcein-AM (green points - live cells). 
of cells in the population after $96 \mathrm{~h}$ of culture increased 10 -fold compared to the first day of culture $(24 \mathrm{~h})$ and 5 -fold compared to the second day of culture ( $48 \mathrm{~h}$ ) (Fig. 3A). It is important to compare the results to the second day of culture, because on this day the cancer cells were introduced into the microsystem and the process of creating the cellular multilayer was started. It was observed that the proliferation of the cells co-cultured under microfluidic conditions was significantly higher than the proliferation of the cancer cells in monoculture. Thanks to this, we proved that the presence of the stromal cells is a stimulating factor which increases the divisions of cancer cells. Additionally, a microscopic analysis (based on differences in the morphology of fibroblasts and cancer cells) confirmed that the population of the non-malignant cells in the microsystem was completely covered by the cancer cells. This indicates the formation of a heterogeneous cellular multilayer.

Studies of the proliferation of A2780 and HOF cell cultures and HOF/A2780 co-culture were also independently performed by flow cytometry. The obtained results are consistent with the results obtained using the AlamarBlue test in a microfluidic system (Fig. S1 and Table 1, ESI†).

\section{Effectiveness of PDT with free and nanoencapsulated meso- tetrafenylporphyrin on the 3D cancer model}

A new cellular model in the form of a cellular multilayer and prepared in the microsystem, was used to evaluate the cytotoxic and photocytotoxic properties of PS: free (TPP) and nanoencapsulated (nano-TPP) form of meso-tetrafenylporphyrin. An effective photosensitizer should be non-toxic in the dark, it should selectively accumulate in the tumor tissue and not cause the phototoxic effects in healthy tissues. ${ }^{44}$ Two forms of PS were tested to compare their effectiveness in the treatment of ovarian cancer (in multilayer culture) and to check whether encapsulation with the use of nanoparticles can improve the properties of PS. The photosensitizers were tested in the concentration range of $0-30 \mu \mathrm{M}$. In the case of the cytotoxicity testing a control sample was the cells untreated with PS and untreated with a light, but in the case of the photocytotoxicity studying a control sample was the irradiated cells without PS (Fig. S3, ESI $\dagger$ ).

Fig. 4 shows the results obtained for free TPP tested on the $\mathrm{HOF} / \mathrm{A} 2780$ cellular multilayer under microfluidic conditions. A decrease in the ovarian cell viability with an increase in the tested drug concentration before (cytotoxicity) and after (photocytotoxicity) cell irradiation was observed. The free form of TPP has cytotoxic properties for ovarian cells for concentrations higher than $3 \mu \mathrm{M}$. For a concentration of $3 \mu \mathrm{M}$, the cell viability after exposure to the drug without irradiation was $83.1 \% \pm 6.4 \%$, while for concentration of $5 \mu \mathrm{M}$, the viability was only $62.4 \% \pm 8.6 \%$. For each concentration higher than $5 \mu \mathrm{M}$, the cell viability was lower than 50\% (Fig. 4A). After irradiation, a further decrease in the cell viability was observed for each concentration of TPP. The obtained results indicated that only a low concentration $(3 \mu \mathrm{M})$ of free TPP can be used in PDT, but the drug at this concentration has a low therapeutic
A

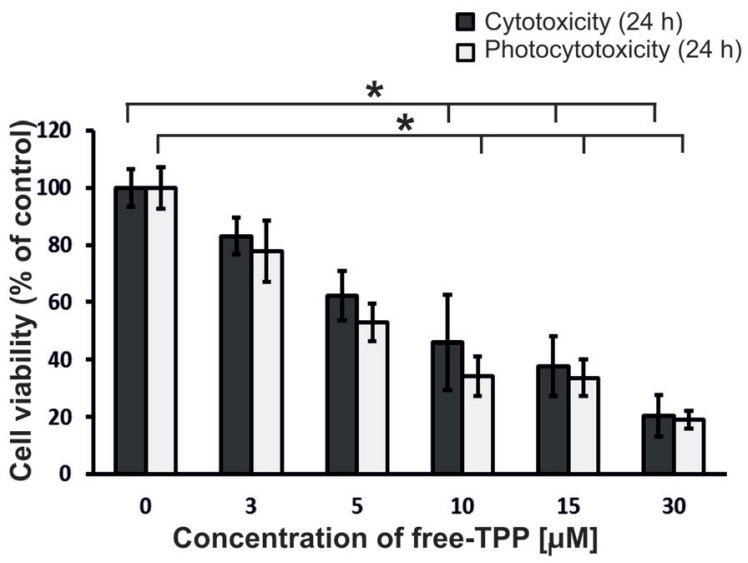

B

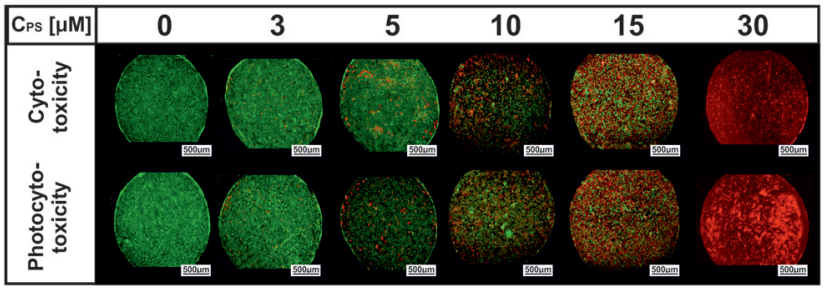

Fig. 4 The viability of multilayered co-culture of HOF/A2780 cells before and after PDT procedure with free TPP. (A) The quantitative results obtained by spectrofluorimetric measurements in the microfluidic system (AlamarBlue test) and (B) the qualitative results obtained by staining cells with fluorescent dyes in a single microchamber (CAM/PI test, green colour - live cells, red colour - dead cells).

efficacy (the cell viability after irradiation was $77.8 \% \pm 10.8 \%$ ). A photosensitizer with good photodynamic properties should have low activity in the dark (without irradiation). ${ }^{45}$ However, we decided to carry out a PDT procedure for all tested drug concentrations to check how encapsulation will affect the cytotoxic and photocytotoxic properties of the drug. The quantitative results obtained in the AlamarBlue test were confirmed by CAM/PI differential staining (Fig. 4B).

Fig. 5 shows the results obtained for nano-TPP, tested on the same cellular model as free TPP. It was observed that nano-TPP at concentrations of $0-5 \mu \mathrm{M}$ did not show cytotoxic properties. The cell viability for these concentrations was around $100 \%$. A low decrease in viability was noticed for $10 \mu \mathrm{M}$ of nano-TPP: the cell viability decreased by $19.4 \% \pm$ $12.8 \%$ of control. The obtained results indicated that nanoTPP in concentrations of $0-10 \mu \mathrm{M}$ can be used in PDT. However, high PS concentrations were cytotoxic and the cell viability equaled $48.5 \% \pm 8.6 \%$ and $35.9 \% \pm 13.5 \%$, respectively for $15 \mu \mathrm{M}$ and $30 \mu \mathrm{M}$. After the PDT procedure, an interesting result was observed for the concentration of $5 \mu \mathrm{M}$ of nano-TPP. The drug used at this concentration was not cytotoxic, but, after irradiation, it had a high therapeutic efficacy, because it caused a decrease in the cell viability by $62.3 \% \pm$ 1.5\% (Fig. 5A). A similar dependence, a significant therapeutic efficacy, was also noticed for $10 \mu \mathrm{M}$ of nano-TPP. The results indicate that the drug can be used in the PDT studies in con- 


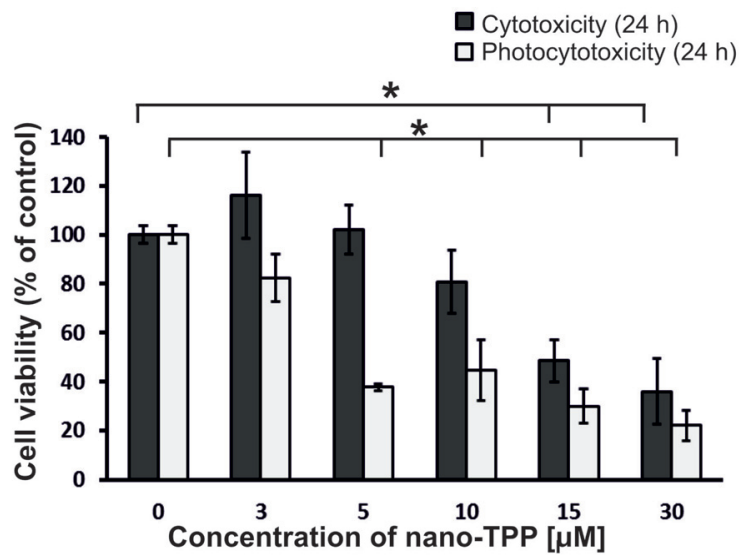

B

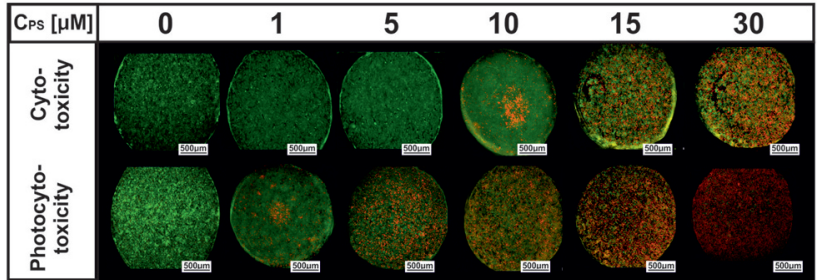

Fig. 5 The viability of multilayered co-culture of HOF/A2780 cells before and after PDT with nano-TPP. (A) The quantitative results obtained by AlamarBlue test and (B) the qualitative results obtained by cells staining (CAM/PI) in a single microchamber.

centration of $10 \mu \mathrm{M}$. The obtained results were confirmed in an independent CAM/PI test (Fig. 5B).

The results of cyto- and photocytotoxicity tests carried out on the $3 \mathrm{D}$ cancer model in the microfluidic microsystem for TPP and nano-TPP confirmed our hypothesis that encapsulation affects the properties of the photosensitizer. Polyelectrolyte nanocapsules made it possible to limit the cytotoxic properties of the PS. ${ }^{26,46}$ Thanks to the encapsulation process, it is possible to extend the range of concentrations of the tested PS in the application in PDT to $10 \mu \mathrm{M}$.

Additional cytotoxicity and photocytotoxicity tests were performed using nano-TPP $(0 \mu \mathrm{M}-15 \mu \mathrm{M})$ in the microsystem. The studies were conducted independently on the monoculture of both HOF and A2780 cells (Fig. 6). Thanks to this, we could check whether the nanoencapsulated PS is selective for cancer
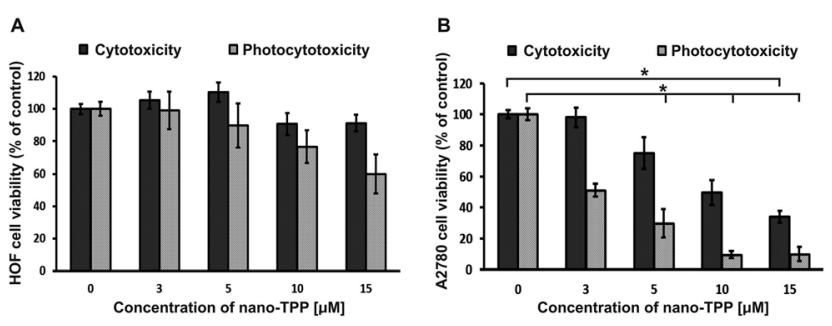

Fig. 6 (A) The viability of non-malignant cells and (B) cancer cells cultured as monolayers under microfluidic conditions before and after PDT procedure with nano-TPP. cells. We could compare the results obtained in the monocultures to the results obtained on the cellular multilayer. The obtained results confirmed that nano-TPP is not cytotoxic and photocytotoxic to ovarian fibroblasts (HOF cells) (Fig. 6A). Based on these observations, we conclude that encapsulation of the PS may promote the selective accumulation of the drug in cancer cells. In addition, in the case of the tests performed on the cancer cell monolayer (Fig. 6B), a very high photocytotoxic effect was observed. Such an effect was not observed in the studies performed on the cellular multilayer. Cellular monolayers were directly exposed to the compound and irradiated afterwards, which probably caused the drug to have too strong of an effect. Interactions between stroma and cancer cells in co-culture may affect the metabolism of the photosensitizer. ${ }^{11}$ It is most likely that in the described case, the results of weakening the drug or inducing cancer cells' resistance by the stromal cells were observed. Finally, the structure of the 3D tissue is more resistant to the effects of treatment.

The effectiveness of photodynamic therapy using nano-TPP under macroscale conditions was also tested. Comparison of results obtained using standard $2 \mathrm{D}$ culture methods with results obtained in the microsystem has been presented in ESI (Fig. S2†).

\section{The level of reactive oxygen species (ROS) in HOF/A2780 cells before and after PDT}

The reactive oxygen species (ROS) are formed by the presence of molecular oxygen and are characterized by high chemical reactivity. Under physiological conditions, ROS production is strictly controlled by the organism, because these particles have highly destructive properties. ${ }^{47}$ The ROS cause chemical modifications and damage to proteins, lipids, carbohydrates, nucleotides (which lead to changes in the DNA). The ROS generated during the PDT procedure can directly kill cancer cells by initiating apoptosis or necrosis. The ROS may also damage the DNA of cancer cells ${ }^{48}$ or lead to the degradation of the blood vessels in a tumor. ${ }^{49}$ Cell death then occurs due to hypoxia of the tumor tissue.

The level of ROS generated in ovarian cells was reviewed to check the photodynamic therapy mechanism with the nanoencapsulated photosensitizer (nano-TPP). The level of ROS was assessed before and after the PDT procedure in the non-malignant HOF and cancer (A2780) cell monocultures, as well as in the co-culture of two cell lines (HOF/A2780) (Fig. 7). Based on previous results (Fig. 5), we determined that for the studies of the ROS level, we would use a concentration of $5 \mu \mathrm{M}$ of nanoTPP, because this concentration showed the best therapeutic effect. The control samples were cells not treated with drugs (0 $\mu \mathrm{M}$ of nano-TPP). In any culture: HOF, A2780, HOF/A2780 no changes were observed in the level of ROS before irradiation (DARK) (relative to the control).

In addition, in the HOF monoculture, no significant changes were observed, also after the irradiation of the cells with PS (LIGHT) (Fig. 7A). In contrast, a significant increase in the ROS level in monoculture A2780 (Fig. 7B) and in co-culture HOF/A2780 after irradiation (Fig. 7C) was observed. 
A

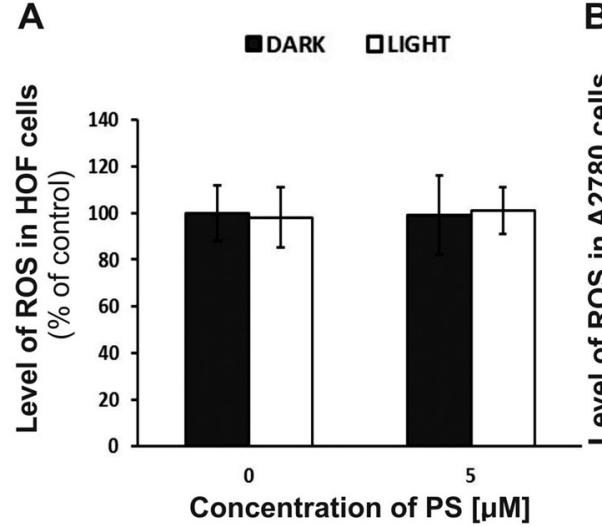

B

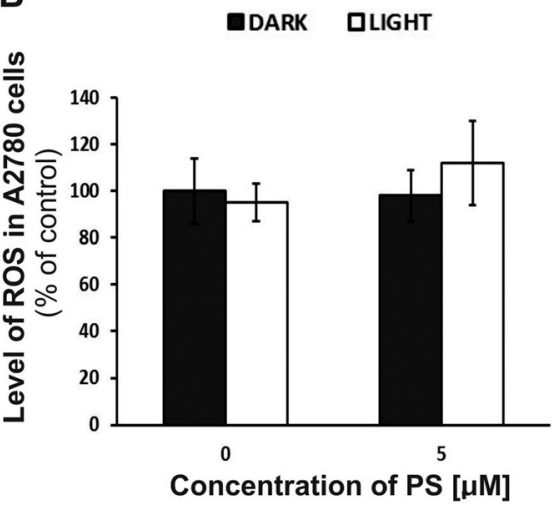

C

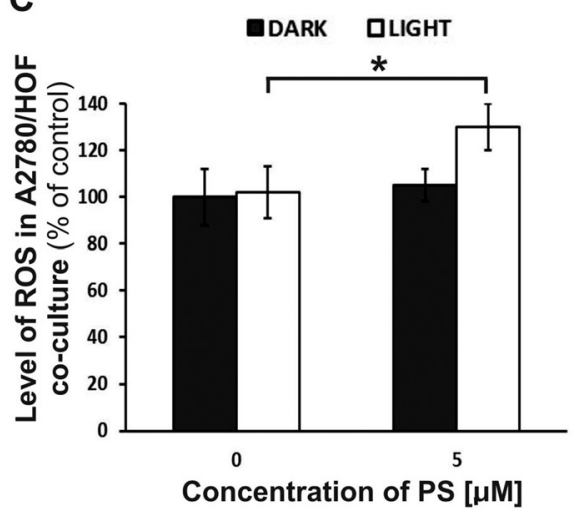

Fig. 7 The ROS level generated in three types of cell culture: (A) HOF monoculture, (B) A2780 monoculture and (C) HOF/A2780 co-culture before and after PDT procedure using nano-TPP as PS in concentration $5 \mu \mathrm{M}$.

The ROS level for monoculture A2780 and co-culture HOF/ A2780 after irradiation was: $112.2 \% \pm 18.1 \%$ and $130.1 \% \pm$ $10.3 \%$, respectively (relative to the control). The obtained results confirmed that the PDT procedure using nano-TPP was carried out in accordance with the theoretical mechanism of therapy. A high level of ROS in the ovarian cell co-culture may be a probable cause of cell death after the PDT procedure.

\section{Discussion}

In this study, we have designed a new perfusion Lab-on-a-chip system for a spatial (3D) cell culture of non-malignant and cancer ovarian cells. We proposed a new 3D cellular model of cancer that can mimic the structure of a fragment of heterogeneous tumor tissue. The developed cancer cellular model has a significant advantage over other 3D cellular models. It mimics the structure of not only the cancer tissue, but also of the cancer stroma. Stroma is very important element of cancer biology and it plays an important role in cancer progression, metastasis or drug resistance. ${ }^{50,51}$ The developed model has the form of a cellular multilayer, that we created thanks to the combination of three important factors: the proper microsystem geometry, the use of microfluidic conditions and a specific combination of the biological material (cell coculture). In the literature, the researchers have tried to develop a cellular multilayer using the microsystem. Montanez-Sauri et al. proposed an automated microfluidic platform, which they used for the spatial culture of breast cancer cells (T47D) in co-culture with human breast fibroblasts (HMF) as a stroma. The presented cell model was used to analyse the protein components of the extracellular matrix (ECM) and to assess the importance of ECM protein composition in the breast cancer progression. ${ }^{52}$ Yan et al. have developed a multilayered microsystem built of the nitrocellulose membranes, on which the breast cancer cells were cultured. The layers of nitrocellulose were placed one of the other, creating a model that mimics the structure of a three-dimensional breast cancer tissue. The researchers did not imitate the cancer stroma, but the cancer flesh only. ${ }^{53}$ Rowan et al. used in their study the adipose tissue-derived stromal cells (ASCs) cultured in coculture with breast cancer cells MDA-MB- $231^{54}$ or head and neck cancer cells Cal-27 and SCC- $4 .{ }^{55}$ In both reports, they proved that the ASCs increase the invasiveness of the cancer cells and stimulate the metastasis. However, in these cases, the stromal cells did not affect the growth of the cancer cells. It is worth noting that these tests were carried out only in static culture conditions.

In the above mentioned examples of the reports, three factors (appropriate geometry, flow, co-culture) which are necessary to create a three-dimensional structure of a fragment of heterogeneous tumor tissue are not always used. A cellular multilayer presented in our work has an advantage over the literature reports, because it imitates the cancer flesh with a layer of the stroma. In addition, the cells arrangement in the multilayer cell culture is vertical and the cells show the high viability in long-term culture.

In our work, we were focused on imitating the cancer ovarian tissue, because ovarian cancer is a significant social problem. This disease is the cause of the death of about 1 million women per year. ${ }^{56}$ Ovarian cancer is often characterized by unknown etiology, high genetic variability, heterogeneity and advanced molecular defense mechanisms, activated against anticancer drugs. ${ }^{57,58}$ High mortality results from the difficulties in diagnostics of ovarian cancer selecting the right method of the treatment is also problematic. Therefore, it is necessary to look for new tools that can facilitate research on the treatment of ovarian cancer.

One of the goals of our work was to check whether the presence of fibroblasts and microfluidic conditions in the culture of the ovarian cancer cells are factors stimulating the progression of this tumor. We confirmed that the stroma contributes to the stimulation of division of the cancer cells cultured in the microfluidic system. In the literature, we can find examples in which the influences of presence of the stromal cells in the cancer cells culture under microfluidic conditions 
was studied. Liu et al. presented the microsystem for coculture the salivary gland cancer cells and carcinoma-associated fibroblasts (CAFs). It was observed that fibroblasts significantly promoted the formation of the cancer cell spheroids and stimulated cell division. These studies confirmed the role of fibroblasts in a tumor invasion. ${ }^{59}$ Jeong et al. created a microsystem for the culture of cellular spheroids made of human colorectal carcinoma cells (HT-29) in co-culture with fibroblasts (CAFs). They proved that in such 3D cellular model, the cancer cells proliferate faster than in monoculture. In addition, the proposed cellular model was useful in the screening of anticancer drugs. ${ }^{60}$

However, it can be noticed, that the majority of similar studies were carried out mainly on cellular spheroids, which mimic the structure of a tumor. So far, the cellular spheroids were the best known 3D tumor model in vitro. Unfortunately, spheroids have some limitations. First, spheroid mimics only the initial stage of tumor growth. ${ }^{61}$ Second, it has a necrotic core made of dead cells because, to spheroid interior, too little of oxygen and nutrients is delivered. ${ }^{62}$ These factors may limit the use of spheroids in studies of anticancer therapies, e.g. in the analysis of the depth of penetration of therapeutic compounds or irradiation.

We hypothesized that the cellular multilayer model of cancer would be suitable for studying the properties of anticancer drugs, for the rapid screening of drug cytotoxicity, and for testing in vitro anticancer therapies. To confirm the hypothesis, we used the ovarian cell multilayer (HOF/A2780) to assess the cyto- and photocytotoxic properties of two photosensitizers: free meso-tetrafenylporphyrin and nanoencapsulated meso-tetrafenylporphyrin as a new-generation drug developed using nanotechnology. We have proven that the process of encapsulation of the tested drug significantly improved its properties as a potential PS for PDT in ovarian cancer treatment. Polyelectrolyte coating reduced the cytotoxicity of the tested drug and did not negatively affect its effectiveness after the PDT procedure. In addition, encapsulation increased the selectivity of the PS, possibly by limiting its accumulation in non-malignant cells. ${ }^{39,46,63}$ The cellular monolayer is the standard research model for testing the efficacy of anti-cancer drugs in vitro. In our studies, we confirmed that multilayered culture of cancer and stromal cells imitates the cancer physiology better than cell monolayer. In the literature, there are only a few examples of similar research conducted by other research groups. Yang et al. proposed a microsystem for creating a fragment of a 3D breast cancer tissue. The authors cocultured the breast cancer cells (MCF-7) with primary adipocytes (ASC) in the form of a multilayer cell culture. The proposed cellular model was successfully applied to study the efficacy of PDT in the treatment of breast cancer. 5-Aminolevulinic acid (5-ALA) was used as a photosensitizer. In addition, gold nanoparticles as a factor supporting the effectiveness of PDT were examined on the model of a fragment of the cancer tissue. ${ }^{13}$ Zuchowska et al. also investigated in microscale the efficacy of PDT on the spheroid culture of the breast cancer cells with human ovarian fibroblasts (MCF-7)
HMF). The nanoencapsulated TPP was used as the PS. The results obtained in this research were convergent with the results obtained on the cellular multilayer, although other cell lines were used in the study. ${ }^{38}$

Although the presented cellular model has many advantages, it also has a few limitations. The arrangement of cells in culture microchambers is not always the same. Our research showed that cells cultured in the microsystem formed threedimensional multilayered regions, but the whole culture in single microchamber did not create a uniform 3D structure (Fig. 2). It is possible to better control the formation of the cellular multilayer in a microsystem, e.g. by changing the microsystem work parameters (e.g., different flow rates, modification of the culture microchamber geometry, reduction of surfacearea-to-volume ratio (SAV). Further research is required to improve the model and obtain a repeatable and uniform $3 \mathrm{D}$ multilayered cell culture.

In our research, we additionally examined the mechanism of action of the nanoencapsulated photosensitizer. We proved that the reactive oxygen species (ROS) are probably responsible for the death of the ovarian cells after the PDT procedure. A high level of ROS in the ovarian cancer cells co-cultured with fibroblasts confirms the utility of the new multilayered cellular model in the PDT study.

\section{Conclusions}

In conclusion, we proved that the multilayer of the non-malignant and cancer cells cultured in the microfluidic system can be a new 3D cellular model of a cancer tissue. Such model is more advanced than other previously known 3D cellular models. The results obtained through the use of a cellular multilayer confirmed that photodynamic therapy can be an effective method of the treatment of ovarian cancer. In addition, the use of the new-generation photosensitizers for PDT can significantly increase the effectiveness of an ovarian cancer treatment and reduce the side effects of an anticancer therapy. A 3D model of the cellular multilayer cultured under microfluidic conditions is similar to a fragment of a heterogeneous tumor tissue in vivo. Thanks to this, it is suitable for the evaluation of cytotoxicity of the new anticancer drugs and the effectiveness of anticancer therapies at the laboratory level. In the future, this model can be used clinically for the rapid selection of effective treatment methods, e.g. in personalized therapy for ovarian cancer.

\section{Conflicts of interest}

There are no conflicts to declare.

\section{Acknowledgements}

This work has been supported by Warsaw University of Technology. The authors wish to thank Prof. D.Sc. Ph.D. Eng. 
Kazimiera A. Wilk and Ph.D. Eng. Urszula Bazylińska from Faculty of Chemistry of Wroclaw University of Science and Technology for synthesis of nanoencapsulated mesotetrafenylporphyrin.

\section{References}

1 F. Zheng, F. Fu, Y. Cheng, C. Wang, Y. Zhao and Z. Gu, Small, 2016, 12, 2253.

2 K. Duval, H. Grover, L. H. Han, Y. Mou, A. F. Pegoraro, J. Fredberg and Z. Chen, Physiology, 2017, 32, 266.

3 L. E. Niklason, Cell Stem Cell, 2018, 22, 302.

4 R. Malik, P. I. Lelkes and E. Cukierman, Trends Biotechnol., 2015, 33, 230.

5 H. Li, X. Fan and J. M. Houghton, J. Cell. Biochem., 2007, 101, 805.

6 Y. Choi, E. Hyun, J. Seo, C. Blundell, H. C. Kim, E. Lee, S. H. Lee, A. Moon, W. K. Moon and D. Huh, Lab Chip, 2015, 15, 3350.

7 O. De Wever and M. Mareel, J. Pathol., 2003, 200, 429.

8 D. W. McMillin, J. Delmore, E. Weisberg, J. M. Negri, D. C. Geer, S. Klippel, N. Mitsiades, R. L. Schlossman, N. C. Munshi, A. L. Kung, J. D. Griffin, P. G. Richardson, K. C. Anderson and C. S. Mitsiades, Nat. Med., 2010, 16, 483.

9 D. W. McMillin, J. M. Negri and C. S. Mitsiades, Nat. Rev. Drug Discovery, 2013, 12, 217.

10 E. S. Nakasone, H. A. Askautrud, T. Kees, J. H. Park, V. Plaks, A. J. Ewald, M. Fein, M. G. Rasch, X. Y. Tan, J. Qiu, J. Park, P. Sinha, M. J. Bissell, E. Frengen, Z. Werb and M. Egeblad, Cancer Cell, 2012, 21, 488.

11 L. Milla Sanabria, M. E. Rodríguez, I. S. Cogno, N. B. Rumie Vittar, M. F. Pansa, M. J. Lamberti and V. A. Rivarola, Biochim. Biophys. Acta, 2013, 1835, 36.

12 X. J. Li, A. V. Valadez and P. Zuo, Bioanalysis, 2012, 4, 1509.

13 H. Ma, T. Liu, J. Qin and B. Lin, Electrophoresis, 2010, 31, 1599.

14 Y. Yang, X. Yang, J. Zou, C. Jia, Y. Hu, H. Du and H. Wang, Lab Chip, 2015, 15, 735.

15 H. Yamazoe, Y. Hagihara and H. Kobayashi, Tissue Eng., Part C, 2016, 22, 20.

16 Z. Xu, Y. Gao, Y. Hao, E. Li, Y. Wang, J. Zhang, W. Wang, Z. Gao and Q. Wang, Biomaterials, 2013, 34, 4109.

17 B. Sebastian and P. S. Dittrich, Annu. Rev. Fluid Mech., 2018, 50, 483.

18 Y. C. Chen, G. Y. Chen, Y. C. Lin and G. J. Wang, Microfluid. Nanofluid., 2010, 9, 585.

19 Q. Tu, L. Pang, Y. Zhang, M. Yuan, J. Wang, D. Wang and W. Liu, Chin. J. Chem., 2013, 31, 304.

20 D. T. Chiu, A. J. deMello, D. Di Carlo, P. S. Doyle, C. Hansen, R. M. Maceiczyk and R. C. R. Wootton, Chem, 2017, 2, 201.

21 A. N. Karnezis, K. R. Cho, C. B. Gilks, C. L. Pearce and D. G. Huntsman, Nat. Rev. Cancer, 2017, 17, 65.
22 H. Azaïs, N. Delhem, C. Frochot, L. Colombeau, A. Grabarz, O. Moralès and P. Collinet, Eur. J. Obstet. Gynecol. Reprod. Biol., 2019, 234, e181.

23 T. Teshigawara, M. Mizuno, T. Ishii, Y. Kitajima, F. Utsumi, J. Sakata, H. Kajiyama, K. Shibata, M. Ishizuka and F. Kikkawa, Photodiagn. Photodyn. Ther., 2018, 21, 121.

24 Y. Matoba, K. Banno, I. Kisu and D. Aoki, Photodiagn. Photodyn. Ther., 2018, 24, 52.

25 B. Mansoori, A. Mohammadi, M. Amin Doustvandi, F. Mohammadnejad, F. Kamari, M. F. Gjerstorff, B. Baradaran and M. R. Hamblin, Photodiagn. Photodyn. Ther., 2019, 26, 395.

26 M. Chudy, K. Tokarska, E. Jastrzebska, M. Bułka, S. Drozdek, Ł. Lamch, K. A. Wilk and Z. Brzózka, Biosens. Bioelectron., 2018, 101, 37.

27 S. S. Lucky, K. C. Soo and Y. Zhang, Chem. Rev., 2015, 115, 1990.

28 B. Bazylińska, R. Frąckowiak, Z. Brzózka and K. A. Wilk, J. Photochem. Photobiol., B, 2017, 166, 169.

29 J. Kulbacka, A. Pucek, M. Kotulska, M. Dubińska-Magiera, J. Rossowska, M. P. Rols and K. A. Wilk, Bioelectrochemistry, 2016, 110, 19.

30 L. Guyon, M. Ascencio, P. Collinet and S. Mordon, Photodiagn. Photodyn. Ther., 2012, 9, 16.

31 K. A. Kujawa and K. M. Lisowska, Postepy Hig. Med. Dosw., 2015, 69, 1275.

32 R. Allison, R. Cuenca, G. Downie, M. Randall, V. Bagnato and C. Sibata, Photodiagn. Photodyn. Ther., 2005, 2, 51.

33 G. Di Lorenzo, G. Ricci, G. M. Severini, F. Romano and S. Biffi, Theranostics, 2018, 8, 4279.

34 N. Shishkova, O. Kuznetsova and T. Berezov, Cancer Biol. Med., 2012, 9, 9.

35 P. Tudrej, K. A. Kujawa, A. J. Cortez and K. M. Lisowska, Diagnostics, 2019, 9, E120.

36 K. Ziółkowska, R. Kwapiszewski and Z. Brzózka, New J. Chem., 2011, 35, 979.

37 E. Tomecka, K. Zukowski, E. Jastrzebska, M. Chudy and Z. Brzozka, Sens. Actuators, B, 2018, 254, 973.

38 A. Zuchowska, K. Marciniak, U. Bazylinska, E. Jastrzebska, K. A. Wilk and Z. Brzozka, Sens. Actuators, B, 2018, 275, 69.

39 M. Flont, E. Jastrzębska and Z. Brzozka, Anal. Chim. Acta, 2020, 1100, 138-148.

40 B. Di Stasio, C. Frochot, D. Dumas, P. Even, J. Zwier, A. Müller, J. Didelon, F. Guillemin, M. L. Viriot and M. Barberi-Heyob, Eur. J. Med. Chem., 2005, 40, 1111.

41 M. Sibrian-Vazquez, T. J. Jensen and M. G. Vicente, J. Photochem. Photobiol., B, 2007, 86, 9.

42 M. Canete, A. Villanueva, V. Dominguez, S. Polo, A. Juarranz and J. C. Stockert, Int. J. Oncol., 1998, 13, 497.

43 D. Hanahan, R. A. Weinberg and S. Francisco, Cell, 2000, 100, 57.

44 S. Kwiatkowski, B. Knap, D. Przystupski, J. Saczko, E. Kędzierska, K. Knap-Czop, J. Kotlińska, O. Michel, K. Kotowski and J. Kulbacka, Biomed. Pharmacother., 2018, 106, 1098. 
45 D. Bechet, P. Couleaud, C. Frochot, M. L. Viriot, F. Guillemin and M. Barberi-Heyob, Trends Biotechnol., 2008, 26, 612.

46 Ł. Lamch, A. Pucek, J. Kulbacka, M. Chudy, E. Jastrzębska, K. Tokarska, M. Bułka, Z. Brzózka and K. A. Wilk, Adv. Colloid Interface Sci., 2018, 261, 62.

47 G. Y. Liou and P. Storz, Free Radical Res., 2010, 44, 479.

48 N. S. Brown and R. Bicknell, Breast Cancer Res., 2001, 3, 323.

49 C. A. Robertson, D. H. Evans and H. Abrahamse, J. Photochem. Photobiol., B, 2009, 96, 1.

50 S. S. Li, C. K. Ip, M. Y. Tang, S. K. Sy, S. Yung, T. M. Chan, M. Yang, H. C. Shum and A. S. Wong, J. Visualized Exp., 2017, 120, e55337.

51 K. M. Bussard, L. Mutkus, K. Stumpf, C. Gomez-Manzano and F. C. Marini, Breast Cancer Res., 2016, 18, 84.

52 S. I. Montanez-Sauri, K. E. Sung, E. Berthier and D. J. Beebe, Integr. Biol., 2013, 5, 631.

53 W. Yan, Q. Zhang, B. Chen, G. T. Liang, W. X. Li, X. M. Zhou and D. Y. Liu, Chin. J. Anal. Chem., 2013, 41, 822 .
54 B. G. Rowan, J. M. Gimble, M. Sheng, M. Anbalagan, R. K. Jones, T. P. Frazier, M. Asher, E. A. Lacayo, P. L. Friedlander, R. Kutner and E. S. Chiu, PLoS One, 2014, 9, e89595.

55 B. G. Rowan, E. A. Lacayo, M. Sheng, M. Anbalagan, J. M. Gimble, R. K. Jones, W. J. Joseph, P. L. Friedlander and E. S. Chiu, Aesthetic Surg. J., 2016, 36, 93.

56 F. Bray, F. J. Ferlay, I. Soerjomataram, R. L. Siegel, L. A. Torre and A. Jemal, CA Cancer J. Clin., 2018, 88, 394.

57 A. M. Karsta and R. Drapkin, J. Oncol., 2010, 2010, 1.

58 E. A. White, H. A. Kenny and E. Lengyel, Adv. Drug Delivery Rev., 2014, 79-80, 184.

59 T. Liu, B. Lin and J. Qin, Lab Chip, 2010, 10, 1671.

60 S. Y. Jeong, J. H. Lee, Y. Shin, S. Chung and H. J. Kuh, PLoS One, 2016, 11, e0159013.

61 S. Nath and G. R. Devi, Pharmacol. Ther., 2016, 163, 94.

62 C. Giverso and L. Preziosi, Int. J. Non Linear Mech., 2019, 108, 20.

63 E. Jastrzębska, U. Bazylińska, M. Bułka, K. Tokarska, M. Chudy, A. Dybko, K. A. Wilk and Z. Brzózka, Biomicrofluidics, 2016, 10, 014116. 